\title{
Annick Laruelle and Federico Valenciano: Voting and collective decision-making
}

Cambridge University Press, Cambridge, 2008

\author{
Ines Lindner
}

Received: 17 July 2010 / Accepted: 20 July 2010 / Published online: 26 August 2010 (C) The Author(s) 2010. This article is published with open access at Springerlink.com

\begin{abstract}
The book 'Voting and Collective Decision Making' by A. Laruelle and F. Valenciano provides a critical revision of the theoretical foundations of collective yes-or-no decisions. It is a study of the theory of bargaining and voting power, revolving around a fundamental question: given a committee, what voting rule should be used?
\end{abstract}

\section{Brief summary of the book and motivation of research}

In a nutshell, the theory and measurement of voting power revolves around the question to what extent a voter is able to control the outcome of a vote. This question is fundamental as it provides the foundation to the question of what voting procedure should be used for a decision-making body when we deal with criteria such as, e.g. egalitarianism. As an example take the European Union where the decision-making processes of the EU institutions should be ideally designed-from an egalitarian point of view-such that every EU citizen has equal voting power. Especially, the several enlargements of the EU have led to a renewal of interest in this topic and heated debates in the scientific community along with controversial academic papers.

The main purpose of Voting and Collective Decision Making by A. Laruelle and F. Valenciano $(\mathrm{L} \& \mathrm{~V})$ is to provide a critical revision of the theoretical foundations of collective yes-or-no decisions as well as a revision of the recommendations when it comes to the design of decision-making procedures. The focus of their approach is to include not only the voting rule ${ }^{1}$ as unique ingredient but to draw a clear

\footnotetext{
${ }^{1} \mathrm{L \& V}$ define a voting rule as a monotonic and proper simple game. In other words, it is a set of winning coalitions which contains the grand coalition and all supersets of winning coalitions. It does not contain the empty set or the complement of a winning coalition (see their Definition 1, p. 5).
}

I. Lindner $(\varangle)$

Department of Econometrics and OR, VU University Amsterdam, De Boelelaan 1105,

Amsterdam, $1081 \mathrm{HV}$, The Netherlands

e-mail: ilindner@feweb.vu.nl 
distinction regarding the environment of the collective decision making bodies. They introduce two different types of committees: the first has solely the capacity to accept or reject proposals submitted to it and is referred to as 'take-it-or-leave-it committees'. In contradistinction, a 'bargaining committee' has the capacity to bargain over feasible agreements.

This book is a fundamental contribution to the theory and measurement of voting and voting power. L\&V's request to specify the type of the committee (environment) in order to clarify the notion 'power' or 'voting power' is certainly justified. Especially illuminating are their findings in chapter 4 on bargaining committees where they extend classical findings of Nash (1950) by game theoretic concepts of bargaining power. The different chapters of this book could be used as teaching material for graduate courses on voting, bargaining or voting power.

Before I comment in detail on the different chapters let me first elaborate in Sect. 2 of this article why a book of this kind represents a fundamental contribution to the literature on voting and voting power. Sections 3, 4 and 5 of this report evaluate their chaps. 3, 4 and 5 in detail.

\section{Brief detour to history and related literature}

The 64-years history of the theory and measurement of voting power has been haunted by a lot of confusion, conceptually as well as with respect to attribution of authorship. In their historical account from 2005, Felsenthal and Machover (F\&M) identify two main phenomena of this unfortunate evolution. First, similar ideas were reinvented several times as many researchers were unaware of previous work. Second, the meaning and implications of basic concepts was widely misunderstood or simply disregarded by scientists for several decades.

The latter aspect is presumably the main reason why the theory and measurement of voting power has failed to achieve wide acceptance beyond scientific specialism-it suffers from a problem of arbitrariness. ${ }^{2}$ Why applying the Penrose-Banzhaf measure $^{3}$ ? Why not the Shapley-Shubik index ${ }^{4}$ or any other power measure? This question should be addressed first when it comes to studying real-world or designed voting systems. Instead, measures were often applied mechanically with little or no attempt to justify a particular choice. Unfortunately, different measures can lead to different results for the same data which damages the standing of this academic discipline due to the aura of ambiguity.

Another unfortunate common practice, when comparing the positions of a given voter $a$ under two alternative decision rules, is to incautiously normalize power measures and work, e.g. with the Penrose-Banzhaf index, the normalized version of the Penrose-Banzhaf measure. This is an error because the index measures a voter's

\footnotetext{
2 For further reasons confining acceptance see the appendix.

3 Penrose (1946) was the first to propose a measure of voting power which later came to be known as 'the (absolute) Banzhaf measure (index). Penrose's pioneering work was widely ignored and his measure independently reinvented by Banzhaf (1965).

4 Following the terminology of Felsenthal and Machover (1998), I reserve the term index of voting power for relative measures. The latter-as in the case of the Shapley-Shubik index-always add up to 1.
} 
relative share in the total power of all voters, which is not fixed. A change of decision rule may therefore lead to a smaller relative share in total sum of the applied power measures with the voter having an increase in absolute power. This misunderstanding was obvious in the heated debates preceding the Lisbon Treaty in 2007. For example, the Penrose-Banzhaf measure of a representative of Poland in the EU Council of Ministers under the Treaty of Nice is 0.03116 as opposed to the higher value of 0.09816 in the Lisbon Treaty. The Penrose-Banzhaf index, however, draws a completely different picture as it drops from 7.4192 to $5.7113 \%$ (for the numbers see Aziz and Leech forthcoming). The normalization of power measures involves great loss of information and its use for different decision rules is inappropriate as it compares cakes of different sizes. In addition, normalization makes the voting scenario sound like a zero-sum game when in fact all voters could profit from a change of the voting rule, e.g. from unanimity to simple majority. Here, normalization would lead to the erroneous conclusion that both are the same since both are symmetric.

The Measurement of Voting Power: Theory and Practice, Problems and Paradoxes by Felsenthal and Machover (F\&M) from 1998 was the first monograph wholly devoted to the theory of a priori voting power. It provided a systematic critical revision of the foundations of traditional voting power theory. The authors introduced a distinction between two different kinds of voting power: 'power as influence' (I-power) and 'power as a prize' (P-power). The former is operationalized as the probability to influence the decision of a voting body, related to 'policy seeking' as the motive of voting. Here, the outcome of a vote is a public good (or public bad) that may benefit (or harm) all the voters. F\&M consider the (absolute) Penrose-Banzhaf measure as the right measure of a priori voting power on the basis of some I-power postulates (see Sect. 7 in Felsenthal and Machover 1998). The second type of power, P-power, is a notion related to 'office seeking'. The vote (decision-making process) determines the winning coalition who in turn distributes a fixed purse among them. P-power measures the expected share of a voting body's member. F\&M consider the Shapley-Shubik index, derived from cooperative game theory, as the most serious known candidate for a measure of this kind (see Comment 7.10.1 in Felsenthal and Machover 1998). ${ }^{5}$ This index was introduced by Shapley and Shubik (1954) who proposed an index of a priori voting power as a direct application of the Shapley value (as introduced in Shapley 1953) to simple (TU) games. ${ }^{6}$

The I/P-power distinction of F\&M provided a first guidance to the question when to apply which power measure to (real-world) decision making bodies.

With the success of this book, however, also came criticism from which I will briefly name two main points:

(1) F\&M consider the Penrose-Banzhaf measure as the only reasonable way to measure I-power by, whereas they consider the Shapley-Shubik index as the most serious candidate for a measure of P-power. This clear cut distinction represents

\footnotetext{
5 They point out, however, that this index violates the added blocker postulate (see their Definition 7.9.8) which, in their opinion, a reasonable index of a priori voting power should satisfy.

6 The success of this article as well as the neglect of Penrose's earlier work has led to the unfortunate view that the theory and measurement of voting power is a branch of cooperative game theory only. For details see Felsenthal and Machover (2005, Sect. 3).
} 
I-power and P-power as disjunctive concepts which led to critique. For example, Napel and Widgren (2004) develop a framework for measuring influence in contexts that involve strategic interaction and require strategic equilibrium analysis of agenda setting, bargaining, etc. Applying this framework to spatial voting in the UN Security Council, Napel and Widgren (2008) assume a one-dimensional convex policy space and demonstrate that the Shapley-Shubik index can serve as a suitable measure for influence (identified with the probability of being critical for the equilibrium policy outcome).

(2) L\&V consider the I/P power distinction as too vague and argue that the question what 'power' or 'voting power' is cannot be sufficiently addressed without specifying the type of the committee. Not only should the voting rule be taken into account but also the environment in which the rule is applied. The authors blame the vagueness in the specification of the voting situation underlying most of the literature on voting power.

As a first approach to include environments of voting, L\&V distinguish between two types of committees: 'take-it-or-leave-it' (tioli) committees and 'bargaining' committees. The proposals that a tioli committee has to vote upon are (i) independent, i.e. there is no room for linking decisions on different proposals which rules out strategic agreements between the voters, (ii) submitted by some external agency and (iii) are not modifiable, i.e. the committee can only accept or reject them. By contrast a bargaining committee (i) deals with different issues over time as in the tioli case but (ii) bargains about each issue in search of an agreement, in which task it is entitled to adjust the proposal. Moreover, (iii) any winning coalition can enforce an agreement and (iv) for each issue a different configuration of preferences emerges in the committee over the set of feasible agreements. They argue that this distinction provides benchmarks for a better understanding of less clear real-world situations.

L\&V's Chap. 1 introduces some notations and terminologies, whereas Chapt. 2 briefly reviews seminal papers and introduces (informally) the distinction between tioli and bargaining committees. I will comment on the remaining Chaps. 3 to 5. For this purpose I give a brief overview of L\&V's findings first.

\section{Take-it-or-leave-it committees vis-à-vis traditional voting theory}

\subsection{One stage games}

L\&V's Chap. 3 is concerned with tioli committees. As the proposal cannot be modified and there is no room for linking decisions on different proposals (independence) there is no room for strategic considerations. The best that any voter can do is to vote 'yes' or 'no' according to his or her preferences. ${ }^{7}$ The authors give as examples a referendum, and an academic committee that decides by vote on whether to admit a student to a programme without capacity constraints. The key insight and LV's main point is that in tioli situations the relevant notions should be success, i.e. the likelihood of being

\footnotetext{
7 This model allows no indifference between acceptance and rejection.
} 
on the winning side-irrespective of whether one's vote is decisive for it or not. ${ }^{8}$ This is different to the traditional voting power theory which uses the likelihood of being decisive (the Penrose-Banzhaf measure) as the relevant quantity. Both approaches, however, are based on the same probabilistic model that each vote configuration is equally likely. This a priori assumption follows traditional voting theory and is justified on normative grounds. It disregards particular personalities or preferences of the voters such that the actual pattern of voting behavior is not taken into account. ${ }^{9}$ In a priori models, the probability of success of a dummy voter ${ }^{10}$ is equal to $1 / 2$, whereas the one of a dictator equals 1 .

$\mathrm{L} \& \mathrm{~V}$ consider the question of the optimal voting rule in a tioli committee from two points of view: egalitarianism and utilitarianism. The former says that an equal treatment should be given to equals, or, if utility functions are given, the same (expected) utility level. The latter principle intends to maximize the sum of voter's utilities. The utility they introduce for this purpose assumes a utility level of zero in case of "no success' (not being on the winning side) and distinguish between 'positive' success and 'negative' success. ${ }^{11}$ Assuming the same (expected) utility preferences for any voter, $\mathrm{L} \& \mathrm{~V}$ show that egalitarianism is implemented by any rule that assigns equal a priori success to each voter. Of course, any symmetric rule satisfies this principle. The utilitarian principle on top determines the optimal quota of the symmetric rule, which varies with the importance given to positive success. The higher the latter the smaller is the quota (with a lower bound of $1 / 2$ in order to avoid non-proper rules).

\subsection{Committees of representatives}

Section 3.8 uses the material developed in the previous subsection to provide theorybased recommendations on the choice of the voting rule for a committee of representatives - which is a special type of a committee. Here, each member acts on behalf of a group of individuals. As an example take the Council of Ministers of the European Union (EU) where EU citizen's participation in decision making is largely indirect since political decisions are made by representative agents elected by citizens. This is a two-stage process in which citizens exercise direct power at the first stage, in electing their representative, whereas in the second stage the power over actual political decisions is exercised only indirectly by means of the representative. Ideally, every European citizen should be treated equally (L\&V's sense they should have equal a

\footnotetext{
8 The measure 'success' goes back to Penrose (1946) who refers to it as the probability of a voter 'being on the winning side'. Instead of success, however, he proposes to work with a measure which is defined by the amount by which his chance of being on the winning side exceeds one half which-multiplied by $1 / 2$ - later came to be known as the (absolute) Banzhaf measure (index).

9 This abstraction is necessary to focus on the effect of the voting rule, disentangled from behavioral effects (for details see e.g. Roth 1988, p. 9).

10 This is a voter who is never decisive, i.e. who's vote never makes any difference to the outcome of a vote.

11 The former measures the utility of a voter being successful when s/he votes 'yes', the latter when voting 'no' (L\&V's equation 25, p.73). The idea is that when rejection of the proposal to be voted upon means maintaining the status quo there may be a bias in either direction. A conservative party, for example, might consider keeping the status quo more important than changes.
} 
priori expected utility or probability of success), whereas the total sum of expected utilities of all citizens should be maximized. The question of this section is a profound question from a normative point of view: given the number of members of a committee of representatives and the sizes of each group represented, what is the optimal voting rule with respect to the two principles of egalitarianism and utilitarianism?

A common simplifying assumption in two-stage models of citizen's participation in modern democratic states is that every representative always follows the majority opinion of his/her group on every issue. In other words, the group decides by simple majority whether the representative should vote 'yes' or 'no'. This assumption is also used by L\&V with which the question of the optimal voting rule boils down to finding an 'optimal' rule for the second stage which decides how the votes of the representatives should be aggregated in order to make a (political) decision.

Traditional voting power theory uses the Penrose-Banzhaf measure (the likelihood to be decisive) as the default voting power approach for this case. ${ }^{12}$ This assumption leads to the classic first square root rule for two-tier voting systems, which states that if each represented group is sufficiently large, each citizen's Penrose-Banzhaf measures are equal (with negligible error) if and only if the one of the representatives are proportional to the square root of the size of the corresponding represented group. This represents an egalitarian solution based on the Penrose-Banzhaf measure without explicitly defining utilities. ${ }^{13}$

L\&V come to a different conclusion than traditional voting power theory. Since they base their analysis on the probability of success rather than the probability of being decisive, their finding is that in a two-tier voting system the egalitarian principle is basically satisfied for any decision rule used by the committee of representatives. By 'basically satisfied' they mean that the a priori probability of success of any individual is approximately $1 / 2$ regardless of the rule since the a priori likelihood of being critical is negligible if the represented group is sufficiently large. Here, success is approximated by the probability of being on the winning side by mere luck-which equals $1 / 2$ in an (a priori) dichotomous voting situation. As a conclusion, any represented individual in a tioli two-tier voting system should in practice be indifferent with respect to the rule in the committee. This is of course a very different conclusion from the one reached by traditional voting power approach, which is based on comparisons in relative terms between very small numbers (the likelihood of being decisive for individuals) which in L\&V's view 'artificially dramatizes differences between individuals' (L\&V, p.85).

The second square root rule of traditional voting power theory is concerned with minimizing the mean 'majority deficit' of a two-tier voting system. This deficit is defined as the deviation of the indirect two-tier decision-making rule from a 'direct democracy' simple majority rule. As an example take the US elections from 2000

\footnotetext{
12 For example, in F\&M terminology legislation is commonly interpreted as a public good which suggests to base the analysis on I-power. Here, any payoff of being on the winning side does not imply 'taking away' any utility of any other voter. To the contrary, the more people are on the winning side the stronger the collective 'yes' statement.

13 It can be shown, however, that the Banzhaf measure equals expected a priori utility assuming a special type of utility function (L\&V, pp. 85-86).
} 
Table 1 Results for a committee of representatives: traditional voting power theory versus the take-it-orleave-it model

\begin{tabular}{|c|c|c|}
\hline & Traditional voting theory ${ }^{a}$ & Take-it-or-leave-it model \\
\hline Egalitarianism & $\begin{array}{l}\text { First square root rule: assign a voting rule such that the } \\
\text { representative's probability of decisiveness, } \Phi_{\mathrm{r}} \text {, is } \\
\text { proportional to the square root of the size of the } \\
\text { represented electorate } n_{r}: \Phi_{\mathrm{r}} \sim \sqrt{n_{\mathrm{r}}}\end{array}$ & $\begin{array}{l}\text { The voting rule } \\
\text { does not matter }\end{array}$ \\
\hline $\begin{array}{l}\text { Majoritarian- } \\
\text { ism/utilitarianism }\end{array}$ & $\begin{array}{l}\text { Second square root rule (majoritarianism): use a } \\
\text { weighted majority rule such that each representative is } \\
\text { assigned to a voting weight } w_{\mathrm{r}} \text { proportional to the } \\
\text { square root of the size of the represented group, } \\
w_{\mathrm{r}} \sim \sqrt{n_{\mathrm{r}}}, \text { and a relative quota close to } 50 \%\end{array}$ & $\begin{array}{l}\text { Utilitarianism } \\
\text { confirms second } \\
\text { square root rule }\end{array}$ \\
\hline
\end{tabular}

${ }^{a}$ Based on the Penrose-Banzhaf measure

where G.W. Bush won the presidential election although a majority of the US voters was in favor of A. Gore. Here, the majority deficit is measured as the size of the majority in favor of Gore minus the number of voters having voted for Bush. In general, in the a priori model of every voting outcome being equally likely, the mean majority deficit of a voting rule is given by the expected majority deficit.

The second square root rule states that in a two-tier voting system the mean majority deficit is minimized (with negligible error) if the committee of representatives uses a weighted majority rule such that each representative is assigned to a voting weight proportional to the square root of the size of the group and a relative quota close to 50\% (see Theorem 3.4.9 in Felsenthal and Machover 1998 and or Theorem 3 in Felsenthal and Machover 1999). Note that the second square root rule recommends voting weights proportional to the square root of the size of the group, whereas the first square root rule assigns to each representative a Penrose-Banzhaf measure proportional to the square root of the size of the group. L\&V confirm the second square root rule, however, based on different grounds: a utilitarian principle applied to a twotier tioli committee. ${ }^{14}$ Table 1 summarizes the findings of $\mathrm{L} \& \mathrm{~V}$ and traditional voting theory for committees of representatives.

The second square root rule confirms in fact the first one, when Penrose's Limit Theorem (PLT) holds. The latter states that under certain conditions and if the distribution of weights is not too skewed (in other words, the ratio of the largest weight to the smallest is not very high), then the relative powers of the voters tend to approximate closely to their respective relative weights. Lindner and Machover 2004; Lindner 2004; Lindner and Owen 2007 provide proofs for some special cases with respect to the Penrose-Banzhaf measure as well as the Shapley-Shubik index. In other words, when PLT holds the voters' voting weights are with close approximation proportional to their Penrose-Banzhaf measures (Shapley-Shubik indices).

When PLT holds, this finding suggests in combination with the first square root rule to assign voting weights to the representatives proportional to the square root of

14 For completeness, it should be stressed that this is the result when positive and negative success are equally important to the individuals. If there is a bias in either direction, L\&V's results suggests that the voting weights stay the same, however, the quota should be adjusted (their Proposition 25 and 26). 
the represented groups (constituencies) ${ }^{15}$ —in accordance with the second square root rule. $^{16}$

\subsection{Take-it-or-leave-it models: critical appraisal and placement in the literature}

As stressed by L\&V on p. 67, success and decisiveness are related. In particular, it can be shown in the a priori case of non-spatial dichotomous voting-where every voting outcome is assumed to be equally likely - the probability of success is given by one half (luck - success without being decisive) plus the product of the likelihood of decisiveness times one half (outcome would change if voter changes his or her mind). The concept of a priori success and decisiveness 'differ only in using a different scale of measurement' (Hosli and Machover 2004). It should be stressed, however, that this only holds in the a priori case where all vote configurations are equally probable. For general probability distributions over vote configurations, 'success and decisiveness are not only conceptually different but also analytically independent' (p. 68).

However, a priori models can still provide different results with respect to the concepts success and decisiveness when it comes to two-tier systems. L\&V show that this is the case for the criteria of egalitarianism when the individual likelihood of being decisive is very small-as in the two-tier EU Council of Ministers case-such that success is approximately given by $1 / 2$, regardless of the rule. Here, the ratio between the success probabilities of two voters is always approximately 1 whereas the relative terms between very small numbers (the likelihood of being decisive for individuals) might not be. However, note that the findings of $\mathrm{L} \& \mathrm{~V}$ does not rule out the first square root rule as it states that with respect to egalitarianism the rule does not matter. In addition, if PLT holds both the traditional and the tioli approach come to the same conclusion (since the second square root rule confirms the first one). In summary, the result of both approaches do not oppose each other.

Whether or not one agrees with this view, L\&V's specification of the environment of voting is without any doubt an important step towards the fundamental issue of what the relevant notions are in the context of voting and how to measure them. However, a tioli committee is a very special case of an environment. L\&V themselves admit that it is seldom found in real-world committees where there is usually some room for negotiations (p. 53). Nevertheless, it provides an important reference point for more realistic models.

Let me close the discussion on Chap. 3 by the following remark: note that the probability of being lucky — to have success without being decisive-depends on the model setup. As an example take voting situations with several ordered levels of approval in the input and in the output, so called $(j, k)$ simple games with $\mathrm{j}$ options (see e.g.

\footnotetext{
15 This suggestion - the so called Jagelionian compromise - played an important role in the heated debates about the reforms of the EU Council of Ministers preceding the Lisbon Treaty (see e.g. http://www. economist.com/world/europe/displaystory.cfm?story_id=9341010). See also Sect. 5 of this article.

16 Note that this result gives an answer to the methodological problem of the first square root rule as to how to find a voting rule such that the Penrose-Banzhaf measures fulfill the proportion requirement. If PLT holds, the Penrose-Banzhaf measures are approximately proportional to the voting weights such that assigning the latter proportional to the square root of the population establishes the egalitarian principle.
} 
Freixas and Zwicker 2003). Here, the voting situation could be a committee having to determine a grade for a student. With $j$ options the likelihood to have the (most) preferred voting outcome by sheer luck is $1 / j$, which approaches zero when the number of options increases. It is clearly zero in spatial voting games as e.g. when the vote is on the preferred tax rate in a continuum between 0 and 1 (see e.g. Napel and Widgren 2009). Here, the a priori probability of success equals the probability of being decisive as getting one's preferred outcome by luck is a priori equal to zero.

\section{Bargaining committees: at the intersection of voting and cooperative game theory}

Chapter 4 of L\&V discusses voting situations in a bargaining environment. This bargaining setup crucially distinguishes itself from the tioli setup in that the proposal can be adjusted in order to reach a consensus among a winning coalition which has the capacity to enforce it. The committee negotiates in search of an agreement over a set of feasible alternatives. The latter is determined by a voting rule that prescribes which coalitions can enforce an agreement. The second ingredient next to the voting rule is the voters' preference profile (the set of feasible utility vectors). L\&V's game theoretic model thus has two basic ingredients: the voting rule and the preference profile. In other words, for a given number of voters a bargaining solution in the L\&V setup is a map from the Cartesian product of all feasible preference profiles and the set of all voting rules to the set of all payoffs vectors in an appropriate Euclidean space.

\subsection{One stage games}

The question of player's justified (expected) payoff is first approached from a cooperative-axiomatic point of view. Given the voting rule and preferences the question is as to what can be considered as reasonable expectations for rational players (the value of players). The two-ingredient model allows L\&V to generalize Nash's bargaining theory which can be seen as the special case where the voting rule is unanimity. For this special case, Nash (1950) provided compelling conditions about the expected outcome: efficiency, symmetry, independence of irrelevant alternatives and invariance with respect to positive affine transformations. He showed that these four conditions determine a unique solution, known as the Nash solution, which is given by the point in the set of feasible utility vectors ${ }^{17}$ where the product of utility gains with respect to the status quo (the disagreement outcome) is maximized. ${ }^{18}$

The symmetry axiom of the Nash solution implies that symmetry between the players' set of feasible utility vectors and status quo results in equal bargaining skills and hence equal payoffs. Kalai (1977) introduced the family of non-symmetric Nash solutions, which is derived by dropping the symmetry axiom and singles out outcomes that maximize the product of utility gains, after each gain is raised to the power of a

\footnotetext{
17 This set is assumed to be compact and convex.

18 In his paper from 1953 he replaces the condition symmetry by anonymity, requiring that the labels of the players should not influence the solution.
} 
player specific weight. These weights can be interpreted as an expression of different bargaining skills (power). ${ }^{19}$

These weights are precisely the impact of the voting rule on the bargaining agreement in the setup of L\&V. This impact is therefore separated from the bargaining solution following Nash in the sense that voting rules affect the bargaining skills by means of the weights in the asymmetric Nash product. By adapting the axioms used by Nash (1950) and two of the axioms used by Shapley (1953) to their two-ingredient setting, L\&V intend to narrow down the set of admissible agreements. ${ }^{20} \mathrm{~L} \& \mathrm{~V}$ 's main result of this chapter (their Theorem 29) shows that these conditions are not enough to single out an agreement for a bargaining problem, however, they restrict the structure of the solution. Their result says that the player's expectations are determined by the weighted Nash solution where the weights are an anonymous real-valued function of the voting rule (not the preference profile), assigning a weight of zero (no bargaining skills) to members occupying null seats. The classical Shapley-Shubik index qualifies as a possible candidate for these weights.

Given a certain voting rule, a further implication of their result is that the bargaining solution for TU-committees ${ }^{21}$ can be taken as weights in the Nash product in order to determine the solution for any configuration of preferences (any utility space). ${ }^{22}$

Next to the axiomatic point of view from cooperative game theory, L\&V approach the question about reasonable agreements by considering the bargaining process from which the axiomatic approach abstracts. In particular, L\&V introduce protocols which set up rules according to which negotiations proceed in the committee. For example, a protocol should state how a player gets into the position to put a proposal for an agreement on the table and what happens if this proposal is not accepted. Here, the decision-making process is modelled as a non-cooperative game. In this approach, the final vote is merely the formal settlement of a non-cooperative process of bargaining.

The positive approach of setting up protocols describing real-world bargaining processes is of course very complex. L\&V's aim is to provide a simple reference model that shows how the given set of winning coalitions could influence this protocol. The main result of this section identifies a stationary ${ }^{23}$ subgame perfect equilibrium where the corresponding payoff vectors converge to the asymmetric Nash bargaining

\footnotetext{
19 Binmore (1998) interpreted these as strategic advantages in specific bargaining circumstances. Grout (1984) uses the asymmetric Nash solution to model bargaining outcomes between unions and shareholders on input levels, profits and wages depending on union bargaining skills.

20 Next to Nash's three other conditions on the payoff space, they adapt the condition 'anonymity' used by Nash-referring to the preference configuration_and Shapley_referring to the voting rule-to hold for both elements. In other words, when players switch their labels in the preference space and the voting game, this should not affect their expected payoff. Finally, the adaptation of Shapley's condition 'null player' says that a player occupying a null seat (who's vote never makes any difference) should be assigned to her status quo payoff.

21 By definition, transferable utility (TU) games are cooperative games where utility can be transferred between players. Hence the only relevant information is a real number - the total utility-for each subset of a coalition. In the special case of simple voting this number is either zero (losing) or one (winning).

22 This is L\&V's equation (55).

23 Here, a strategy of a player consists of (1) a proposal whenever it is his or her turn to be the proposer and (2) a threshold as to what proposals he or she would accept from others. Stationary means that this action plan stays the same in every round.
} 
solution when the probability of failure or breakdown of the process goes to zero (the game always goes on when a proposal is not accepted). Here, the individual weights in the Nash product are given by the probabilities with which a player is chosen to be a proposer (their Theorem 33).

In the following, these exogenous probabilities are specified further by introducing different probabilistic protocols, i.e. assigning concrete probabilities of selecting a coalition and a corresponding proposer. The idea is to link the probability to be the proposer - the source of bargaining power-with the voting rule. Among other indices, the Shapley-Shubik index emerges under a variety of protocols as e.g. Shapley (1953) classical 'protocol' of choosing an order of the players at random and let players join a coalition in this order until a winning coalition is formed. Here, the last pivotal player is the proposer such that his or her overall probability of being the proposer is given by the Shapley-Shubik index. ${ }^{24}$

\subsection{Bargaining in a committee of representatives}

The question of the best voting rule in terms of egalitarianism and utilitarianism is difficult in bargaining committees since these two concepts usually conflict. Egalitarianism would suggest that any player gets equal utility gains in absolute terms with respect to the status quo, whereas utilitarianism suggests to maximize the sum of all utility gains. Shapley (1969) showed that the Nash bargaining solution can be seen as a compromise between these conflicting concepts. ${ }^{25}$

From L\&V's axiomatic cooperative-game-theoretic result we learned that any reasonable bargaining solution with given configuration of preferences and a voting rule should drop out as a solution to Nash's asymmetric bargaining solution where the weights of the Nash product are determined entirely by the voting rule. Assume that we accept the above compromise as 'fair' then then any symmetric voting rule would implement such a compromise since it implies equal weights in the Nash product and hence leads to the classical Nash bargaining solution.

The situation, however, is less obvious when it comes to a committee of representatives in which each member votes on behalf of the represented group which typically have different sizes (as in the Council of Ministers of the EU). 'Mass bargaining' under a symmetric decision rule would be fair, however, this is of course very difficult to realize. The question is now how to find a voting rule for the committee of representatives such that any player would be indifferent between mass bargaining and leaving bargaining in the hands of the representatives. This what L\&V call the 'neutral' rule. Assuming a strong degree of symmetry of preference profiles within each group, ${ }^{26} \mathrm{~L} \& \mathrm{~V}$ show that any voting rule is neutral which implies for each

\footnotetext{
24 As L\&V point out this is equivalent to choosing one of the decisive players in the coalition at random to be the proposer.

25 He showed the existence of weights such that the weighted utility gain (weight times the utility gain) for each player is the same and the sum of the weighted utility gains is maximized. Furthermore, he showed that for these weights the common solution is given by the Nash bargaining solution.

26 In particular, with the payoff of all other players outside one group fixed, the set of feasible payoffs for the players in that group is assumed to be symmetric. Note that the latter covers a large range of possible scenarios from unanimous preferences to zero-sum scenarios within a group.
} 
representative member bargaining power proportional to the size of the represented group (their Theorem 36).

\subsection{Critical appraisal of L\&V's findings on bargaining committees}

In general, the binary decision rule of a dichotomous voting situation formally incorporates the same amount of information as a simple game in the sense of cooperative game theory (in which coalitions either get a 'pie' of size zero or one to be distributed among them). This has contributed to the widespread fallacy that a priori voting power theory is a branch of cooperative game theory only. As pointed out by Felsenthal and Machover (2005), the spread of this mistaken view was further encouraged by a false or at best misleading statement of Shapley and Shubik who claimed that '... any scheme for imputing power among the members of a committee system either yields the power index defined above or leads to logical inconsistency.' (1954, p. 789). As F\&M point out, the problem with this claim is that it refers to the uniqueness proof of the Shapley value from Shapley's 1953 article, however, this proof is defined for the larger class of all cooperative games. The Shapley-Shubik index is the Shapley value on the subclass of simple games. This subclass is not closed under the algebraic operation of game addition - a feature entering the proof of uniqueness of the Shapley value. '..but why should readers - particularly non-mathematicians - suspect this, given the great authority of Shapley and Shubik?' (Felsenthal and Machover 2005, p. 7).

As mentioned earlier the Shapley-Shubik index of a voter can be interpreted as a likelihood of the voter being decisive (pivotal) in a probabilistic queue bargaining model. ${ }^{27}$ Shapley (1953) himself never claimed that the probabilistic queue bargaining model was convincingly realistic but mentioned it after the axiomatic work had been done. F\&M presume that his purpose to introduce this model was to provide "primarily a mathematical artefact: it may not be genuinely realistic, but it is a mathematically well-defined model that yields the Shapley value' as well as 'a useful visualization and a formal aid in reasoning about the Shapley value. For example, it provides an immediate guarantee that it behaves mathematically as expectations (in the probabilistic sense) of the players' respective shares in a fixed total prize.' (Felsenthal and Machover 1998, p. 183). Nevertheless, the interpretation of this index as a probability of turning the balance much like the Penrose-Banzhaf measure contributed to the misunderstanding that both are of the same kind.

The two-ingredient model of L\&V carefully distinguishes between the voting rule and the associated simple TU game and therefore rules out any confusion. The Shapley-Shubik index now clearly drops out as the size of pieces of 'cake' as a measure of voting power: it is a rational expectation in a bargaining situation with TU-like preference profile. Furthermore, L\&V's two-ingredient setup covers all classical bargaining problems and all simple TU games associated with a voting rule. Note that this covers 'power as a price' following the notion of Felsenthal and Machover (1998)

\footnotetext{
27 Here, an order of the players is chosen at random. Starting with the first player, the other players join in this order until a winning coalition is formed. The last player joining is called 'pivotal'.
} 
as a special case where the vote (decision-making process) determines the winning coalition who in turn distributes a fixed purse among them (a simple TU game).

In addition, L\&V's non-cooperative approach allows the interpretation of bargaining power in a precise game-theoretic sense. Here, the source of power is the likelihood of being the proposer given by the protocol. ${ }^{28}$

A shortcoming of L\&V's axiomatic setup is that it fails to single out one particular bargaining agreement (bargaining power index) as a reasonable candidate. The finding is that the Shapley-Shubik index is just one candidate fulfilling compelling requirements on a bargaining solution as a function of the preferences and the voting rule. This index drops out as the unique candidate by adding Dubey (1975) transfer axiom (L\&V's Theorem 31) defined on the voting rule. This axiom, however, has come under critique for a lack of a compelling interpretation. ${ }^{29}$

When it comes to bargaining in a committee of representatives, $L \& V$ showed that a fair voting rule for this committee would be one that assigns each member bargaining power proportional to the size of the represented groups. However, due to the problem that several indices qualify for measuring bargaining power, this result leaves open as to which index should be applied. Here, the non-cooperative results of associating the Nash weights with different bargaining protocols could lead to a particular choice, say, the Shapley-Shubik index which drops out when the protocol could be interpreted as one of the decisive players in the coalition at random to be the proposer (after the wining coalition has been formed by adding one player at random starting from the empty coalition).

\section{Application to the EU Council of Ministers}

It is a fundamental principle of the EU that all citizens should have equal rights in whatever country they happen to live. This egalitarian idea implies that the voting rule should be designed such that any European citizen should have equal 'voting power' (equal expected utility, respectively). The heated debates on how to reform the Treaty of Nice ${ }^{30}$ showed how the problem of arbitrariness-which power measure to choose - can be used in a manipulative way. For example, the Penrose-Banzhaf index (the normalized Penrose-Banzhaf measure) of a representative from Poland in the Council of Ministers (CM) drops from 7.4192 to $5.7113 \%$ when the Treaty of Nice

\footnotetext{
28 Note that in the L\&V's general setup of a probabilistic protocol, a proposal has to be accepted by all players. The authors justify it by concern about 'seeking consensus' (p. 119). However, it would be interesting to see whether or how the results change when this assumption is not made.

29 This axiom was originally formulated as 'additivity' in Shapley (1953) on the class of all cooperative games. Here, leaving alone whether it is compelling, it has at least a clear meaning saying that the sum of the values of a player in two games should be the same as his value of the sum of the games. The subclass of simple games, however, is not closed under the algebraic operation of game addition. Therefore Dubey (1975) modified 'additivity' to 'transfer' in order to provide a uniqueness statement for a value on the class of simple games. Its interpretation, however, remains questionable.

30 The main critique on the Treaty of Nice was that (1) the threshold agreed on is set too high for the Council to be an effective democratic decision-making body, (2) the decision rule (triple majority) is not transparent and difficult to extend when more countries join, (3) the EU members are not adequately represented. For details see Felsenthal and Machover (2001) and Leech (2002).
} 
is replaced by the Lisbon Treaty ${ }^{31}$ which seemed to justify the vigorous Polish protest. These numbers, however, are misleading as they disregard two important issues. (1) It is inappropriate to compare cakes of different sizes - the unnormalized PenroseBanzhaf measure gives a different picture. Here, by replacing the Treaty of Nice with the Lisbon Treaty, every country wins in terms of influence. (2) The Treaty of Nice is not a good reference point as the distribution of 'voting power' was skewed to begin with. For example, the ratio between the Penrose-Banzhaf measure of a Polish citizen and that of a German citizen was 1,4. Hence when moving towards more fairness we cannot help the fact that overrepresented countries loose in a relative sense.

In the lively debates on the reforms on the Treaty of Nice in 2007, the Jagelionain principle - also referred to as 'the square root rule'-was favored by a large part of the scientific voting society (except L\&V) and vigorously supported by the Polish president at that time, Lech Kaczynski, who had even declared his readiness to 'die for the square root'. ${ }^{32}$ The Jagelionain principle suggests to assign voting weights to the representatives proportional to the square root of the population. This principle is deduced from the first and second square root rule (see Table 1), together with the assumption the relative powers of the voters tend to approximate closely to their respective relative weights (which is correct when Penrose's Limit Theorem ${ }^{33}$ holds).

Next to the voting weights, a vital part of the Jagelonian compromise is the optimal quota: it is the one for which the relative voting powers of the voters tend to be most closely proportional to their respective weights. For the 27-member EU, it is well above one half. ${ }^{34}$ A correct formulation for this quota can be found in Machover (2007, p.4).

Chapter 5 of L\&V applies the findings of Chaps. 3 and 4 to the CM of the EU. The authors consider voting rules which were applied from 1958 to 2005 as well as the Convention rule and the Constitution rule. ${ }^{35}$ The latter were discussed as alternatives to the rules of the Treaty of Nice, applied in 2005 to 25 members.

The greatest number of issues in EU parlance is decided by a rule known as qualified majority voting. ${ }^{36}$ From 1958 to 1995 , this has been a weighted voting game (modified with each enlargement) such that each board member is assigned a nonnegative number as weight and a proposed act is adopted if the combined weight of those affirming it achieves a fixed quota. This changed with the Treaty of Nice 2000 whose rules were applied first for 25 members. Here, qualified majority is no longer a single-dimensional weighted decision rule but also contains the additional requirement

\footnotetext{
31 For the data in this passage, see Aziz and Leech (forthcoming).

32 See e.g. http://www.spiegel.de/international/europe/0, 1518,489510,00.html.

33 See Sect. 3.1 of this article.

34 In the limit, when the number of members goes to infinity, this optimal quota tends to $1 / 2$, in agreement with the PLT (see Lindner and Machover 2004).

35 The Constitutional rule is now also known as the Lisbon rule as it was the one selected for the CM in the Treaty of Lisbon 2007. This rule will come into force in 2014 and will be applied on more issues that have been decided upon by unanimity so far.

36 Examples for exceptions include issues concerned with the constitution of the EU itself.
} 
of a winning coalition comprising the majority of members (at least 13 members). ${ }^{37}$ In an attempt to reform the Treaty of Nice in 2001, the Convention rule for 25 members suggests a qualified majority of the majority of the members (13 with 25 members), representing at least $60 \%$ of the population. In the reform that finally was picked up in the writing of the Constitutional Treaty, a winning coalition was described by a coalition that either represented at least $65 \%$ of the population in addition to at least $55 \%{ }^{38}$ of the member states or comprised 22 members. Since L\&V's analysis covers voting scenarios only until 2005, the Jagelionain Compromise is not discussed.

In order to apply the L\&V's findings of their Chaps. 3 and 4 one has to gauge whether the Council acts more like a tioli committee or a bargaining committee. The former is a very special case of an environment. $L \& V$ themselves admit that it is seldom found in real-world committees where there is usually some room for negotiations. ${ }^{39}$ Its special assumptions are certainly not matched when it comes to the two-tier EU system. Especially at the level of the representatives, all kind of strategic behavior and bargaining takes place. As L\&V note themselves, people with experience in working for the EU Council of Minister made it clear that bargaining was a (if not 'the') crucial ingredient in the workings of the Council. This view is supported by the observation that in EU practice a formal vote only takes place when unanimity can be expected. ${ }^{40}$ For this purpose, the proposal to be voted on is modified following the negotiations of the CM members.

On the other hand, L\&V point out that with the further enlargements of the EU, informal bargaining might get more difficult which suggests forfeiting the concern about general consensus. In this case, they argue, their measures developed for tioli committees could provide an interesting assessment. In particular, they consider the ease of passing proposals ${ }^{41}$, individual probability of success as well as their conditional variants. For a normative a priori evaluation of voting rules they follow the usual a priori model that assigns equal probability to each possible coalition, keeping in mind, however, that implications based on this model have no predictive power.

\section{Critical appraisal of L\&V's Chap. 5}

The authors do not give theory-based advice as to what the 'optimal' rule would be for the CM. None of their discussed models 'captures the complexity of the real situation'

\footnotetext{
37 The Treaty of Nice also contained suggestions for further prospective enlargements which came into practice in 2007 when Bulgaria and Romania joined.

38 The motivation of the percentages statement was that these rules can also be applied to expansions of the EU.

39 See L\&V (2008, p. 53).

40 The fact that formal vote takes place once the Council has found a unanimous agreement would suggest that effective voting rule is in fact unanimity. On the other hand, with each enlargement of the EU it becomes obvious that 'weights do matter because negotiators know that they can be outvoted' (quote of D. Galloway, an experienced EU practitioner, see L\&V 2008, p. 163). This suggests that different (bargaining) powers still play a role with respect to the final agreement.

41 This is defined as the ratio of all winning coalitions and all possible coalitions. This measure was introduced by Coleman (1971) as 'the power of a collectivity to act'.
} 
(p. 136). Unfortunately, this does not allow to compare their findings with the one of traditional voting theory. On the other hand, however, it stresses the difficulty of constructing 'realistic' models that cover all aspects of decision-making. Even if one agrees on categorizing one issue to be voted on to make the committee more tioli-like or bargaining-like there are different aspects of the procedure diluting this clear-cut definition. For example, the CM votes on proposals submitted by the Commission and can only modify them by common accord, i.e. unanimity. Unless this is the case it is restricted to either accept the proposal or confirm the status quo by refusing it. This clearly is a tioli aspect. On the other hand, unanimous agreement on an amended version of the proposal involves bargaining. Note that this is the official version of a $\mathrm{CM}$ voting procedure.

Based on insiders' account this is how the CM actually works. The Commission starts with an initial proposed act. It then informally and discreetly canvasses the opinions of the members of the CM, and they also negotiate among themselves. If it is found (unofficially) that the proposed act will not be approved by the CM, the Commission amends the proposal and tries again. This is repeated several times. Each such step is very much like an informal vote, in which the proposed act is rejected. In the end, a version of the act is reached which is assured a majority in the CM. Then a formal vote is taken and of course the act is adopted. However, this final vote is a charade. Since a positive outcome is at this stage a foregone conclusion, members only vote against it if they wish to make a political point-mainly in front of their domestic public. Otherwise they vote for the act (even if initially they are not so happy about it) or at worst abstain (which is tantamount to voting 'no', but is a face-saving way of not being seen too clearly to have lost).

One could argue that what we have here is a series of informal tioli votes with negative outcomes, followed by a theatrical official vote with positive outcome. On the other hand, the Commission amends the proposal until a majority can be expected. These amendments incorporate the negotiation outcomes among the CM members. This is clearly a bargaining aspect. With successive enlargement of the EU, however, informal bargaining becomes more and more difficult such that bargaining might decline.

In conclusion, the official as well as the actual procedure covers tioli as well as bargaining aspects. Hence the clear cut distinction between the two types of committees is not straightforward in the case of the CM of EU. Although the concepts are helpful to understand impacts of the voting rules on the working of the Council, they are not settling the issue of CM voting analysis and voting rule design. It is of course not necessary to squeeze EU decision-making either in a tioli or a bargaining corset. By definition, both concepts for committees are mutually exclusive, however, they are not collectively exhaustive such that other measures especially taylored to the CM might be more helpful. A step in this direction could be to follow L\&V's claim to (further) specify the environment of voting into the model. Since qualified majority voting only applies to legislation on which the European Commission or the European Parliament also has a say, one would think of an approach which explicitly models the Council's interaction and negotiation with these institutions, in particular including the latter as players. Napel and Widgren (2009) consider such an explicit model of the 
EU decision-making process (carrying out strategic equilibrium analysis of the EU's consultation procedure in a spatial model).

As an alternative to the model of Napel and Widgren, one could specify L\&V's noncooperative approach of a bargaining committee for EU decision-making. ${ }^{42}$ Recall that their approach considers protocols setting up rules according to which negotiations proceed in the committee. Here, the motivation was to single out one particular candidate of the set of rational bargaining agreements (characterized by L\&V's Theorem 29). A protocol tailored to EU proceedings could perform the task to choose the appropriate bargaining power measure for EU applications.

Based on L\&V's results on tioli and bargaining committees, one could still make the careful attempt to deduce an 'optimal' voting rule for two-tier voting systems like the CM. Since the authors claim that from an egalitarian point of view the rule does not matter anyway in two-tier tioli committees, the collective decision could be governed entirely from an egalitarian point of view of a bargaining model. Here, with respect to the represented EU citizens, the rule should be a weighted rule that assigns any representative bargaining power proportional to the size of the country represented (L\&V's Theorem 36). ${ }^{43}$ Recall that in bargaining committees this finding was considered to be a compromise between utilitarian and egalitarian aspects in two-stage games. Finally, the remaining degrees of freedom-e.g. if several voting rules or different quota qualify_ could be used following utilitarian criteria for tioli committees.

\section{Appendix}

Arbitrariness is not the only reason why the acceptance of the theory and measurement of voting and voting power is still modest. Further-probably minor reasons next to ambiguity - are (1) the critique that a priori voting power is said to misrepresent actual voting power as well as (2) computational problems.

(1) A priori voting power abstracts away from voters' preferences, the degree of affinity between the voters and coalition formation as well as the prior bias with respect to the issue to be voted upon. The point is to provide insight into the power implications of the decision rule itself rather than on other aspects of the political environment. Roth (1988, p.9) puts it this way. 'This kind of analysis seems to be just what is needed to analyze the voting rules in a new constitution, for example, long before the specific issues to be voted on arise or the specific factions and personalitites that will be involved can be indentified.' Nevertheless, a priori voting power is often said to misrepresent actual voting power, a criticism to which I would like to retort as follows. (i) Any discussion on a posteriori or actual voting power (as opposed to a priori) cannot go without understanding the power equipment due to the voting rule itself, i.e. a priori voting power. (ii) Measures of actual voting power are typically based on real-world frequencies of observed voting patterns over a suitable period of time. These frequencies

\footnotetext{
42 See L\&V 2008, Subsect. 4.4.

43 In order to achieve that, the European commission could be made redundant as an agenda setter.
} 
are then taken to estimate the probability of occurrence of every possible voting configuration. Leaving aside the thorny question of how to obtain sufficiently reliable information of such kind ${ }^{44}$ this approach is not without problems. As an example take the problem of strategic voting when voter's misreport their true preferences in order to manipulate the voting outcome to their favor. ${ }^{45}$ Dichotomous monotonic voting rules, however, preclude the problem of strategic voting.

(2) When it comes to actually compute the value of a (power) measure for a given decision-making body, two difficulties are typical, getting worse with increasing number of voters: running time and memory requirements. This stems from the fact that the voters face the binary decision to vote 'yes' or 'no', which implies that the total number of possible outcomes rises exponentially with increasing assembly size. Already with a modest number of voters, say 27 as is the case in the EU, the problem might be beyond any disposable computational capacity (see Leech 2003 and Lindner 2004). For weighted voting games, the computational effort can be reduced in order to increase polynomially using the method of generating functions combined with storing methods for sparse matrices (Lindner 2004). Unfortunately, this method is not without limitation due to another algorithmic complexity which is memory requirement (space complexity). This problem can be substantial, both in terms of integer size and array dimension which asks for approximation methods (see Leech 2003; Lindner 2004).

Open Access This article is distributed under the terms of the Creative Commons Attribution Noncommercial License which permits any noncommercial use, distribution, and reproduction in any medium, provided the original author(s) and source are credited.

\section{References}

Aziz H, Leech D (forthcoming) The double majority voting rule of the EU Reform Treaty as a democratic ideal for an enlarging union: an appraisal using voting power analysis. In: Słomczyński W, Życzkowski K (eds) Distribution of power and voting procedures in the European Union, Ashgate

Banzhaf JF (1965) Weighted voting doesn't work: a mathematical analysis. Rutgers Law Rev 19:317-343 Binmore KG (1998) Game theory and the social contract II, just playing. MIT Press, Cambridge

Coleman JS (1971) Control of collectivities and the power of a collectivity to act. In: Lieberman B (ed) Social Choice. Gordon and Breach, New York. Reprinted in Coleman JS (1986), Individual interests and collective action, Cambridge University Press, Cambridge

Dubey P (1975) On the uniqueness of the Shapley value. Int J Game Theory 4:131-139

Felsenthal DS, Machover M (1998) The measurement of voting power: theory and practice, problems and paradoxes. Edward Elgar, London

\footnotetext{
${ }^{44}$ For example, in the EU's CM, a vote held is usually the result of a foregoing bargaining process: before the formal vote is taken there is usually a whole series of 'shadow votes' and the official vote only takes place when a majority can be expected. In addition, the Council seems to publish only positive outcomes, i.e. when acts have been adopted.

45 As an example take the 2008 US president election when the battle about the Democratic presidential nomination between Clinton and Obama threatened to harm the chances of the Democrats. Allegedly many US voters in favor of the Republican's candidate McCain reported voted for Clinton in order to keep the harmful Democratic battle alive.
} 
Felsenthal DS, Machover M (1999) Minimizing the mean majority deficit: the second square-root rule. Math Soc Sci 37:25-37

Felsenthal DS, Machover M (2001) The Treaty of Nice and Qualified Majority Voting. Soc Choice Welf 18:431-464

Felsenthal DS, Machover M (2005) Voting power measurement: a story of misreinvention. Soc Choice Welf 25(2-3):485-506

Freixas J, Zwicker W (2003) Weighted voting, abstention, and multiple levels of approval. Soc Choice Welf 21:399-431

Grout PA (1984) Investment and wages in the absence of binding contracts: a Nash bargaining approach. Econometrica 52:449-460

Hosli MO, Machover M (2004) The Nice Treaty and voting rules in the Council: a reply to Moberg (2002). J Common Market Stud 42:497-521

Kalai E (1977) Nonsymmetric Nash solutions and replication of 2-person bargaining. Int J Game Theory 6:129-133

Leech D (2002) Designing the voting system for the Council of the European Union. Public Choice 113: 437-464

Leech D (2003) Computing power indices for large voting games. Manag Sci 49(6):831-838

Lindner I (2004) Power measures in large weighted voting games-asymptotic properties and numerical methods. PhD thesis, University of Hamburg, 2004. Available at http://www.sub.uni-hamburg.de/ opus/volltexte/2004/2222/pdf/Dissertation.pdf

Lindner I, Machover M (2004) L.S. Penrose's limit theorem: proof of some special cases. Math Soc Sci 47(1):37-49

Lindner I, Owen G (2007) Cases where the Penrose Limit Theorem does not hold. Math Soc Sci 53:232-238

Machover M (2007) Penrose's square-root rule and the EU Council of Ministers: significance of the quota. In: Distribution of power and voting procedures in the EU, 12-13 October 2007, European Centre Natolin, Warsaw, Poland. (Unpublished). Available at: http://eprints.1se.ac.uk/2857/

Napel S, Widgren M (2004) Power measurement as sensitivity analysis. J Theor Polit 16(4):517-538

Napel S, Widgren M (2008) Shapley-Shubik versus strategic power-live from the UN Security Council. In: Braham M, Steffen F (eds) Power, freedom and voting. Springer Verlag, Berlin, pp 99-118

Napel S, Widgren M (2009) Strategic vs. non-strategic voting power in the EU Council of Ministers: the consultation procedure, Working paper

Nash JF (1950) The bargaining problem. Econometrica 18:155-162

Nash JF (1953) Two-person cooperative games. Econometrica 21:128-140

Penrose L (1946) The elementary statistics of majority voting. J R Stat Soc 109:53-57

Roth AE (1988) Introduction to the Shapley value. In: Roth AE (ed) The Shapley value. Cambridge University Press, Cambridge

Shapley LS (1953) A value for $n$-person games. In: Kuhn HW, Tucker AW (eds) Contributions to the theory of games II, (Annals of Mathematics Studies 28). Princeton University Press, Princeton

Shapley LS, Shubik M (1954) A method for evaluating the distribution of power in a committee system. Am Polit Sci Rev 48:787-792

Shapley LS (1969) Utility comparison and the theory of games. In: La Décision: Agrégation et Dynamique des Ordres de Préférence. CNRS, Paris, pp 251-263 